PROCEEDINGS OF THE

AMERICAN MATHEMATICAL SOCIETY

Volume 128, Number 10, Pages 3043-3046

S 0002-9939(00)05367-3

Article electronically published on April 7, 2000

\title{
SOME COMPARISONS FOR GAUSSIAN PROCESSES
}

\author{
RICHARD A. VITALE
}

(Communicated by Stanley Sawyer)

\begin{abstract}
Extensions and variants are given for the well-known comparison principle for Gaussian processes based on ordering by pairwise distance.
\end{abstract}

\section{IntroduCtion}

Among the most important tools for Gaussian processes are comparison principles, or simply comparisons. Typically they provide for the derivation of upper bounds by majorizing a given process with a second process that is larger in some sense as well as having more tractable properties. For general discussions, see Adler 1], Fernique 4], Ledoux and Talagrand [9, and Lifshits [10. The purpose of this note is to elaborate some variants of perhaps the most widely applied comparison:

Theorem 1. Suppose that $\left\{X_{i}, i \in I\right\}$ and $\left\{Y_{i}, i \in I\right\}$ are two mean-zero Gaussian processes indexed by the same denumerable set $I$, and suppose that

$$
E\left(X_{i}-X_{j}\right)^{2} \leq E\left(Y_{i}-Y_{j}\right)^{2}
$$

for all $i, j \in I$. Then for any non-decreasing, convex $g: \mathbb{R}_{+} \rightarrow \mathbb{R}^{1}$,

$$
E g\left(\sup _{i, j}\left(X_{i}-X_{j}\right)\right) \leq E g\left(\sup _{i, j}\left(Y_{i}-Y_{j}\right)\right)
$$

and

$$
E \sup _{i} X_{i} \leq E \sup _{i} Y_{i} .
$$

This is associated with Sudakov [14, [15] and Fernique [3] with a later proof by Alexander [2] (Ledoux and Talagrand also mention unpublished work of S. Chevet). Important extensions and variants have been given by Gordon [5], 6], [7] and Kahane 8 .

We wish to show a sequence of extensions that are apparently new. In the sequel, $\left\{X_{i}, i \in I\right\}$ and $\left\{Y_{i}, i \in I\right\}$ continue to be mean-zero Gaussian processes indexed by a denumerable $\boldsymbol{I}$ and satisfying (1); $\left\{m_{i}, i \in I\right\}$ are arbitrary constants. The first extension is

$$
E \sup _{i}\left\{X_{i}+m_{i}\right\} \leq E \sup _{i}\left\{Y_{i}+m_{i}\right\} .
$$

Received by the editors October 12, 1998 and, in revised form, November 12, 1998.

1991 Mathematics Subject Classification. Primary 60G15; Secondary 60E15.

(C)2000 American Mathematical Society 
This implies that, for any constant $m$ and $k \in I$,

$$
E \sup _{i}\left\{X_{i}+m_{i}-X_{k}, m\right\} \leq E \sup _{i}\left\{Y_{i}+m_{i}-Y_{k}, m\right\} .
$$

Finally, for any non-decreasing, convex $g: \mathbb{R}^{1} \rightarrow \mathbb{R}^{1}$ with (i) $g(-\infty)>-\infty$ or with

(ii) $\max \left[E g_{+}\left(\sup _{i}\left\{X_{i}+m_{i}\right\}-X_{k}\right), E g_{+}\left(\sup _{i}\left\{Y_{i}+m_{i}\right\}-Y_{k}\right)\right]<\infty$, one has

$$
E g\left(\sup _{i}\left\{X_{i}+m_{i}\right\}-X_{k}\right) \leq E g\left(\sup _{i}\left\{Y_{i}+m_{i}\right\}-Y_{k}\right) .
$$

In the next section, we provide proofs. The third section shows how a special case is related to a set of weakened Slepian-Schläfli assumptions.

\section{Proofs}

We use the fact that any positive constant can be regarded as nearly the supremum of a centered Gaussian process (cf. Vitale [16]). A technical formulation and proof are as follows:

Lemma 1. For any $c \geq 0$, there is a sequence $\left\{W_{j}\right\}_{j=1}^{\infty}$ of mean-zero Gaussian variables such that almost surely (i) $c \leq \sup _{j \geq 1}<\infty$ and (ii) $\sup _{j \geq n} W_{j} \downarrow c$ as $n \rightarrow \infty$.

Proof. By homogeneity, it is enough to consider $c=1$. Referring to an example of Marcus and Shepp [11], one has the following: for independent, standard Gaussian variables $\left\{Z_{j}\right\}_{1}^{\infty}$ and $\left\{\sigma_{j}\right\}_{1}^{\infty}$ with $1 / \sigma_{j}^{2}=2 \log j+2 \log \log j$, let $W_{1}=\sigma_{1} Z_{1}, W_{2}=$ $-W_{1}, W_{3}=\sigma_{2} Z_{2}, W_{4}=-W_{3}, \cdots$. Then $P\left(\sup _{j \geq 1} W_{j} \geq 1\right)=1$ and $\sup _{j \geq 1} W_{j}$ has an atom at 1 . In fact, the required verifications for these two properties show that they depend only on the tail behavior of the sequence $\left\{\sigma_{j}\right\}$. Hence, more generally, $P\left(\sup _{j \geq n} W_{j} \geq 1\right)=1$ and $\sup _{j \geq n} W_{j}$ has an atom at 1. A standard $0-1$ argument using the tail measurability of $\lim \sup _{j} W_{j}$ then yields the required convergence.

Proofs of (4), (5), (6). To establish (4), it suffices to consider $I$ finite, say $I=\{1,2, \ldots, N\}$, since the general case follows from the Monotone Convergence Theorem; then one may also assume that $0 \leq \min _{i} m_{i}$ since adding a constant to each side of (4) amounts to a shifted set of $m_{i}$. Finally, without loss of generality and using the lemma, one may assume that the underlying probability space is rich enough to support mean-zero Gaussian variables $\left\{W_{i j}\right\}$ that are independent of the $\left\{X_{i}, Y_{i}\right\}$, and such that for each $1 \leq i \leq N, \sup _{j \geq n} W_{i j} \geq m_{i}$ and $\sup _{j \geq n} W_{i j} \downarrow m_{i}$ as $n \rightarrow \infty$.

Consider

$$
\sup _{i}\left\{X_{i}+\sup _{j \geq n} W_{i j}\right\}=\sup _{i, j \geq n}\left\{X_{i}+W_{i j}\right\} .
$$

A similar expression for the $Y$ process can also be written as a supremum of meanzero Gaussian variables. It can be checked that the two collections of variables are ordered according to (1). Consequently, (3) implies

$$
E \sup _{i}\left\{X_{i}+\sup _{j \geq n} W_{i j}\right\} \leq E \sup _{i}\left\{Y_{i}+\sup _{j \geq n} W_{i j}\right\} .
$$

Each integrand is bounded from below by 0 and is decreasing in $n$, so that letting $n \rightarrow \infty$ and applying the Dominated Convergence Theorem then yields (4). 
For (5), set $\hat{X}_{i}=X_{i}-X_{k}$ and $\hat{Y}_{i}=Y_{i}-Y_{k}$ for $i \neq k$ with $\hat{X}_{k}=\hat{Y}_{k}=0$; further set $\hat{m}_{i}=m_{i}-m_{k}$ for $i \neq k$ and $\hat{m}_{k}=\max \left\{m, m_{k}\right\}$. Then (4) holds for the hatted system, and this is equivalent to $(5)$.

For (6), assume as before that $I$ is finite and $g(-\infty)>-\infty$. From (5), the following holds for $-\infty<t<\infty$ :

$$
E \sup _{i}\left\{X_{i}+m_{i}-X_{k}, t\right\} \leq E \sup _{i}\left\{Y_{i}+m_{i}-Y_{k}, t\right\} .
$$

Subtracting $t$ leads to the equivalent form

$$
E g_{t}\left(\sup _{i}\left\{X_{i}+m_{i}-X_{k}\right\}\right) \leq E g_{t}\left(\sup _{i}\left\{Y_{i}+m_{i}-Y_{k}\right\}\right)
$$

where $g_{t}: \mathbb{R}^{1} \rightarrow \mathbb{R}^{1}$ is given by $g_{t}(\cdot)=(\cdot-t)_{+}$. Then it is enough to recall that the closed, positive linear hull of the collection $\left\{g_{t}\right\}$ is precisely the class of nonnegative, non-decreasing, convex functions on $\mathbb{R}^{1}$; adding a constant adjusts for a given limit at $-\infty$.

For the alternate assumption, note that the truncation $\max \{g, c\}$ conforms to the previous requirement and that the truncation can be removed by letting $c \rightarrow-\infty$ and appealing to the Monotone Convergence Theorem.

\section{A CONnECtion With the Slepian-SchläFli COMPARISON}

The well-known Slepian-Schläfli comparison ([12, [13]) gives a stronger statement than (3) at the expense of a more stringent hypothesis. It can be formulated as follows:

Suppose that, together with (1), one has for all $i \in I$

$$
E X_{i}^{2}=E Y_{i}^{2} \text {. }
$$

Then for all $t>0$

$$
P\left(\sup _{i} X_{i}>t\right) \leq P\left(\sup _{i} Y_{i}>t\right) .
$$

Unfortunately, the requirement of strict equality in (8) often precludes applicability. While there seems to be no easy remedy for this, let us show that our earlier results can be adapted to a weak form of (8), which leads in turn to an integrated form of (9):

$$
\int_{t}^{\infty} P\left(\sup _{i} X_{i}>s\right) d s \leq \int_{t}^{\infty} P\left(\sup _{i} Y_{i}>s\right) d s
$$

for all $t>0$. In place of $(8)$, consider

$$
E X_{i}^{2} \leq E Y_{i}^{2}
$$

for all $i \in I$. Then, assuming (1) and (11), we have

$$
E \sup _{i}\left\{X_{i}+m_{i}, m\right\} \leq E \sup _{i}\left\{Y_{i}+m_{i}, m\right\}
$$

and for any non-decreasing, convex $g: \mathbb{R}^{1} \rightarrow \mathbb{R}^{1}$ with (i) $g(-\infty)>-\infty$ or with (ii) $\max \left\{E g_{+}\left(\sup _{i}\left\{X_{i}+m_{i}\right\}\right), E g_{+}\left(\sup _{i}\left\{Y_{i}+m_{i}\right\}\right)\right\}<\infty$,

$$
E g\left(\sup _{i}\left\{X_{i}+m_{i}\right\}\right) \leq E g\left(\sup _{i}\left\{Y_{i}+m_{i}\right\}\right) .
$$


These can be argued as follows, where it suffices once again to assume that $I=$ $\{1,2, \cdots, N\}$. Consider the augmented process $\left\{X_{1}, X_{2}, \cdots, X_{N}\right\} \cup\left\{X_{0}=0\right\}$. Having assumed (1) and (11) for the original process corresponds precisely to (1) holding for the augmented process. We then choose $k=0$, and let $m_{0}=-\infty$ so that $X_{i}+\left.m_{i}\right|_{i=0}=m_{0}$ and $Y_{i}+\left.m_{i}\right|_{i=0}=m_{0}$ do not participate in any suprema. Then (5) and (6) translate to (12) and (13), the analogue of (4) reducing to a special case of (13).

Finally, (10) follows from (13):

$$
\begin{aligned}
\int_{t}^{\infty} P\left(\sup _{i} X_{i}>s\right) d s & =E\left(\sup _{i} X_{i}-t\right)_{+} \leq E\left(\sup _{i} Y_{i}-t\right)_{+} \\
& =\int_{t}^{\infty} P\left(\sup _{i} Y_{i}>s\right) d s .
\end{aligned}
$$

\section{ACKNOWLEDGMENT}

The referee and the Probability Editor provided helpful comments.

\section{REFERENCES}

[1] Adler, R.J. (1990). An Introduction to Continuity, Extrema, and Related Topics for General Gaussian Processes. Institute of Mathematical Statistics. MR 92g:60053

[2] Alexander, R. (1985). Lipschitzian mappings and total mean curvature of polyhedral surfaces I. Trans. Amer. Math. Soc. 288, 661-678 MR 86c:52004

[3] Fernique, X. (1975). Regularité des trajectoires des fonctions aléatoires gaussiennes. Lecture Notes in Mathematics 480, 1-96, Springer. MR 54:1355

[4] Fernique, X. (1997). Fonctions aléatoires gaussiennes vecteurs aléatoires gaussiens. CRM, Montreal. CMP 98:02

[5] Gordon, Y. (1985). Some inequalities for Gaussian processes and applications. Israel J. Math. 50, 265-289. MR 87f:60058

[6] Gordon, Y. (1987). Elliptically contoured distributions. Prob. Th. Rel. Fields 76, 429-438. MR 88m:60042

[7] Gordon, Y. (1992). Majorization of Gaussian processes and geometric applications. Prob. Th. Rel. Fields 91, 251-267. MR 93a:60059

[8] Kahane, J-P. (1986). Une inegalité du type de Slepian et Gordon sur les processus gaussiens. Israel J. Math. 55, 109-110. MR 88a:60075

[9] Ledoux, M. and Talagrand, M. (1985). Probability in Banach Spaces. Springer, New York.

[10] Lifshits, M.A. (1995). Gaussian Random Functions. Kluwer, Boston. MR 98k:60059

[11] Marcus, M. and Shepp, L. (1972). Sample behavior of Gaussian processses. Proc. Sixth Berkeley Symp. Math. Stat. Prob. 2, 423-441. MR 53:6710]

[12] Schläfli, L. (1858). On the multiple integral whose limits are $p_{1}=a_{1} x+b_{1} y+\cdots+h_{1} z>$ $0, \cdots, p_{n}>0, x^{2}+y^{2}+\cdots+z^{2}<1$. Quart. J. Math. Pure Appl. 2, 261-301, also, (1860) 3, $54-68$.

[13] Slepian, D. (1962). The one-sided barrier problem for Gaussian processes. Bell System Tech. J. 41, 463-501. MR 24:A3017

[14] Sudakov, V.N. (1971). Gaussian random processes and measures of solid angles in Hilbert space. Dokl. Akad. Nauk. SSR 197, 43-45.; English translation in Soviet Math. Dokl. (1971) 12, 412-415. MR 44:6027

[15] Sudakov, V.N. (1976). Geometric Problems in the Theory of Infinite-Dimensional Probability Distributions. Trud. Mat. Inst. Steklov 141. English translation in Proc. Steklov Inst. Math 2, Amer. Math. Soc. MR 80e:60052

[16] Vitale, R.A. (1996). Covariance identities for normal random variables via convex polytopes. Stat. Prob. Letters 30, 363-368. MR 98c:62103

Department of Statistics, U-120, University of Connecticut, Storrs, Connecticut 06269-3120

E-mail address: rvitale@uconnvm.uconn.edu 